\title{
Correction to: Functional Capacity Evaluation in Different Societal Contexts: Results of a Multicountry Study
}

\author{
Jone Ansuategui Echeita ${ }^{1}$ Matthias Bethge ${ }^{2}$. Berry J. van Holland ${ }^{3} \cdot$ Douglas P. Gross $^{4} \cdot$ Jan Kool $^{5} \cdot$ Peter Oesch $^{5}$. \\ Maurizio A. Trippolini ${ }^{6,7,8}$. Elizabeth Chapman ${ }^{9} \cdot$ Andy S. K. Cheng $^{10} \cdot$ Robert Sellars $^{11} \cdot$ Megan Spavins $^{12}$. \\ Marco Streibelt ${ }^{13} \cdot$ Peter van der Wurff ${ }^{14,15} \cdot$ Michiel F. Reneman $^{1}$
}

Published online: 26 June 2018

(c) The Author(s) 2018

\section{Correction to: Journal of Occupational Rehabilitation https://doi.org/10.1007/s10926-018-9782-x}

The original version of this article unfortunately contained a mistake in Table 2. The data under column head "Left handgrip strength $(\mathrm{n}=336)$ " was erroneously omitted during the production process. The corrected Table 2 is given below.

The original article has been corrected.
The original article can be found online at https://doi.org/10.1007/ s10926-018-9782-x.

Jone Ansuategui Echeita

j.ansuategui.echeita@umcg.nl

1 Department of Rehabilitation Medicine, University Medical Center Groningen, University of Groningen, P.O. Box 30.002, 9750 RA Haren, Groningen, The Netherlands

2 Institute of Social Medicine and Epidemiology, University of Lübeck, Lübeck, Germany

3 Institute for Sports Studies, Hanze University of Applied Sciences, Groningen, The Netherlands

4 Department of Physical Therapy, University of Alberta, Edmonton, AB, Canada

5 Rehabilitation Centre Valens, Valens, Switzerland

6 Center for Disability Research, Liberty Mutual Research Institute for Safety, Hopkinton, Boston, USA

$7 \quad \mathrm{PhD}$ in Rehabilitation Sciences Program, Institute for Health Professions, Massachusetts General Hospital (MGH), Charlestown, Boston, USA
Open Access This article is distributed under the terms of the Creative Commons Attribution 4.0 International License (http://creativeco mmons.org/licenses/by/4.0/), which permits unrestricted use, distribution, and reproduction in any medium, provided you give appropriate credit to the original author(s) and the source, provide a link to the Creative Commons license, and indicate if changes were made.
8 Department of Work Rehabilitation, Rehaklinik Bellikon, Suva Care, Bellikon, Switzerland

9 Toyota Motor Manufacturing Canada, Puslinch, ON, Canada

10 Ergonomics and Human Performance Laboratory, Department of Rehabilitation Sciences, The Hong Kong Polytechnic University, Hong Kong, China

11 FCE Systems Ltd, Geraldine, New Zealand

12 Occupational Therapy Inc., Randburg, South Africa

13 Department of Rehabilitation, German Federal Pension Insurance, Berlin, Germany

14 Research \& Development, Military Rehabilitation Center Aardenburg, Doorn, The Netherlands

15 Institute for Human Movement Studies, HU University of Applied Sciences, Utrecht, The Netherlands 
Table 2 Results from multiple multilevel regression analyses with FCE test performances as dependent variables are given; unstandardized regression coefficients and their standard errors $(\mathrm{b}(\mathrm{SE}))$, and model's deviance $(-2 *$ LogLikelihood) by the addition of random and fixed effects are shown

\begin{tabular}{|c|c|c|c|c|c|c|c|c|}
\hline & \multicolumn{2}{|c|}{ Floor-to-waist lift ${ }^{\mathrm{a}}(\mathrm{n}=294)$} & \multicolumn{2}{|c|}{ Six minute walk $^{\mathrm{b}}(\mathrm{n}=224)$} & \multicolumn{2}{|c|}{ Right handgrip strength ${ }^{\mathrm{c}}(\mathrm{n}=335)$} & \multicolumn{2}{|c|}{ Left handgrip strength ${ }^{\mathrm{c}}(\mathrm{n}=336)$} \\
\hline & $\mathrm{b}(\mathrm{SE})$ & Deviance & $\mathrm{b}(\mathrm{SE})$ & Deviance & $\mathrm{b}(\mathrm{SE})$ & Deviance & $\mathrm{b}(\mathrm{SE})$ & Deviance \\
\hline Null model & & 2213.26 & & 2878.28 & & 2847.88 & & 2826.82 \\
\hline Random effects & & $2193.04 * * *$ & & $2867.71 * *$ & & $2806.11 * * *$ & & $2787.86 * * *$ \\
\hline \multicolumn{9}{|c|}{ Clinician and measurement country } \\
\hline Fixed effects $^{\mathrm{d}}$ & & $1941.21 * * *$ & & $2643.19 * * *$ & & $2590.43 * * *$ & & $2597.43^{* * *}$ \\
\hline Intercept & $-2.01(9.86)$ & & $219.87(130.89)$ & & $-3.67(18.00)$ & & $-18.96(17.39)$ & \\
\hline Age (years) & & & & & $-0.16(0.06)$ & & $-0.13(0.05)$ & \\
\hline \multicolumn{9}{|l|}{ Sex } \\
\hline Female & $-4.97(1.01)$ & & & & $-11.33(1.82)$ & & $-11.38(1.77)$ & \\
\hline Height $(\mathrm{cm})$ & $0.17(0.05)$ & & $1.98(0.67)$ & & $0.34(0.10)$ & & $0.38(0.09)$ & \\
\hline BMI $\left(\mathrm{kg} / \mathrm{m}^{2}\right)$ & & & $-3.82(1.16)$ & & & & & \\
\hline \multicolumn{9}{|l|}{ Affected body area } \\
\hline Lower extremity & & & & & $-0.33(2.01)$ & & & \\
\hline Upper extremity & & & & & $-8.19(2.32)$ & & & \\
\hline Neck & & & & & $-5.94(2.02)$ & & & \\
\hline Generalized & & & & & $-5.62(1.82)$ & & & \\
\hline Other & & & & & $9.17(8.30)$ & & & \\
\hline \multicolumn{9}{|l|}{ Observed physical effort } \\
\hline Heavy & $7.74(1.28)$ & & & & & & & \\
\hline Maximum & $9.04(1.70)$ & & & & & & & \\
\hline \multicolumn{9}{|l|}{ Test ended prematurely } \\
\hline Yes & $-4.87(1.19)$ & & $-168.60(24.50)$ & & & & & \\
\hline Post-test HR (bpm) & & & $1.99(0.30)$ & & & & & \\
\hline $\begin{array}{l}\text { Reported pain intensity } \\
\text { (NRS) }\end{array}$ & $-0.64(0.21)$ & & $-10.89(2.81)$ & & $-0.77(0.33)$ & & $-0.91(0.28)$ & \\
\hline $\begin{array}{l}\text { Reported effort dur- } \\
\text { ing FCE test (Borg } \\
\text { CR-10) }\end{array}$ & $-0.61(0.20)$ & & $-20.49(2.79)$ & & & & & \\
\hline Reported social isolation & $-0.34(0.17)$ & & & & & & & \\
\hline $\begin{array}{l}\text { Reported catastrophiz- } \\
\text { ing }\end{array}$ & & & & & $-0.61(0.27)$ & & & \\
\hline $\begin{array}{l}\text { Reported disability } \\
\text { (PDI) }\end{array}$ & $-0.07(0.04)$ & & & & & & & \\
\hline \multicolumn{9}{|c|}{ Physical work demands (DOT) } \\
\hline Light & & & & & $0.65(2.38)$ & & $0.93(2.31)$ & \\
\hline Medium & & & & & $-0.06(2.24)$ & & $0.78(2.19)$ & \\
\hline Heavy & & & & & $6.77(2.51)$ & & $6.26(2.43)$ & \\
\hline Very heavy & & & & & $2.66(2.86)$ & & $5.65(2.77)$ & \\
\hline \multicolumn{9}{|l|}{ Days off work } \\
\hline Less than $1 / 4$ year & & & $3.57(22.30)$ & & & & & \\
\hline $1 / 4-1 / 2$ year & & & $-59.66(22.75)$ & & & & & \\
\hline $1 / 2-1$ year & & & $-12.31(22.18)$ & & & & & \\
\hline $1-2$ years & & & $-27.40(25.08)$ & & & & & \\
\hline More than 2 years & & & $-89.19(24.17)$ & & & & & \\
\hline
\end{tabular}

Reference category: patient's affected body area: low back; observed physical effort by clinicians: light to moderate; patient's physical work demands: sedentary; patient's days off work: no days off

BMI Body Mass Index, HR heart-rate, NRS Numeric Rating Scale, PDI Pain Disability Index, DOT Dictionary of Occupational Titles, FCE Functional Capacity Evaluation

Significance: $*<0.05 ; * *<0.01 ; * * *<0.001$

${ }^{a}$ Measured in $\mathrm{kg}$

${ }^{\mathrm{b}}$ Measured in $\mathrm{m}$

${ }^{\mathrm{c}}$ Measured in $\mathrm{kgF}$

${ }^{\mathrm{d}}$ Each of the fixed effects factors showed a significant improvement of the model at its addition 